\title{
Antioxidant Strategies in Neurocritical Care
}

\author{
Khalid A. Hanafy • Magdy H. Selim
}

Published online: 2 December 2011

(C) The American Society for Experimental NeuroTherapeutics, Inc. 2011

\begin{abstract}
An increase in oxidative stress and overproduction of oxidizing reactive species plays an important role in the pathophysiology of several conditions encountered in the neurocritical care setting including: ischemic and hemorrhagic strokes, traumatic brain injury, acute respiratory distress syndrome, sepsis, and organ failure. The presence of oxidative stress in these conditions is supported by a large body of preclinical and clinical studies, and provides a rationale to support a potential therapeutic role for antioxidants. The purpose of this article is to briefly review the basic mechanisms and molecular biology of oxidative stress, summarize its role in critically ill neurological patients, and review available data regarding the potential role of antioxidant strategies in neurocritical care and future directions.
\end{abstract}

Keywords Oxidative stress · Antioxidants · Neurological . Stroke $\cdot$ Hemorrhage

\section{Introduction}

Increased production of oxidizing free radical species with subsequent oxidative stress (OS) plays an important role in critically ill neurological patients and is central to the pathophysiology of many conditions, such as ischemic and

\section{K. A. Hanafy}

Department of Neurology, Divisions of Neurocritical Care, Beth Israel Deaconess Medical Center,

Boston, MA 02215, USA

M. H. Selim $(\bowtie)$

Department of Neurology, Stroke Division,

Beth Israel Deaconess Medical Center,

330 Brookline Avenue - Palmer 127,

Boston, MA 02215, USA

e-mail: mselim@bidmc.harvard.edu hemorrhagic strokes, traumatic brain injury, subarachnoid hemorrhage, sepsis, acute respiratory distress syndrome, and organ failure. Some studies support an association between increased OS and poor outcomes in the critically ill, suggesting a potential role for antioxidant strategies in neurocritical care [1]. In this article, we summarize the basic mechanisms of OS and its role in critically ill neurological patients, and we review available data regarding the potential utility of antioxidant strategies in the Neuro Intensive Care Unit.

\section{The Basic Mechanisms and Molecular Biology of Oxidative Stress}

Normal physiological processes, such as cellular respiration, generate small amounts of oxidizing reactive oxygen species (ROS) and reactive nitrogen species (RNS). These are chemical highly reactive molecules that can damage cell structures. However, there is an endogenous defense (scavenger) system to protect tissues from ROS- and RNS-induced injury. Scavenger systems include: enzymes, such as superoxide dismutase (SOD), catalase, and glutathione peroxidase; their cofactors (selenium, zinc, copper, iron, and manganese), and sulfhydryl group donors (glutathione, which is involved in amino acid transport across cell membrane and in protein degradation); and nonenzymatic antioxidants, such as vitamins $\mathrm{E}$ and $\mathrm{C}, \beta$-carotene, and hemebinding proteins, such as ceruloplasmin, transferrin, albumin, and haptoglobin $[2,3]$. Oxidative stress refers to an imbalance between the production of ROS and nitric oxide synthase (NOS), and their effective removal by these endogenous protective antioxidants and scavenger enzymes.

In critical illness, there is increased production of ROS and RNS, increased consumption with subsequent reduced stores of antioxidants, and decreased activity of scavenger enzymes $[4,5]$. The circulating levels of antioxidants decrease rapidly 
after critical illness, trauma, or surgery, and remain low for days to weeks [5]. Low levels of antioxidants are associated with increased generation of ROS and RNS, OS, subsequent cell death, and increased morbidity and mortality are among the critically ill $[6,7]$. The brain is particularly vulnerable to OS-mediated injury because of its high oxidative metabolic activity and oxygen consumption with high production of ROS, low antioxidant capacity, high content of polyunsaturated fatty acids and regional iron, and high surface-tomembrane ratio [8].

Increased production of ROS and RNS during critical illness may be related to $\mathrm{Ca}^{++}$activation of phospholipase leading to intracellular accumulation of $\mathrm{Ca}^{++}$and excitotoxicity; activation of NOS and production of nitric oxide (NO); activation of phagocytes, release of iron, copper, and metalloproteins; conversion of xanthine dehydrogenase to xanthine oxidase; activation of eicosanoid pathway and inflammatory response; release of cytokines and expression of adhesion molecules nuclear factor-kappaB (NF-kB) and mitochondrial failure [2, 8-10]. High levels of ROS and intracellular $\mathrm{Ca}^{++}$overload can lead to structural alteration of the mitochondria and electron transport chain, resulting in further increase in ROS generation, and ROS reacts with fatty acids leading to lipid peroxidation and cell membrane damage. Peroxynitrite, the main derivative of nitric oxide, has the ability to nitrate phenyl moieties within tyrosine, changing the conformation of the protein and inducing oxidative damage of proteins and lipids, depression of mitochondrial enzymes, depletion of glutathione, and DNA damage [11]. Figure 1 summarizes the basic mechanisms of OS and pathways for ROS and RNS generation.

\section{Monitoring Oxidative Stress In Vivo}

The currently available methods to assess OS in vivo include: direct quantification by electron spin resonance; or indirect determination of total radical-trapping antioxidant

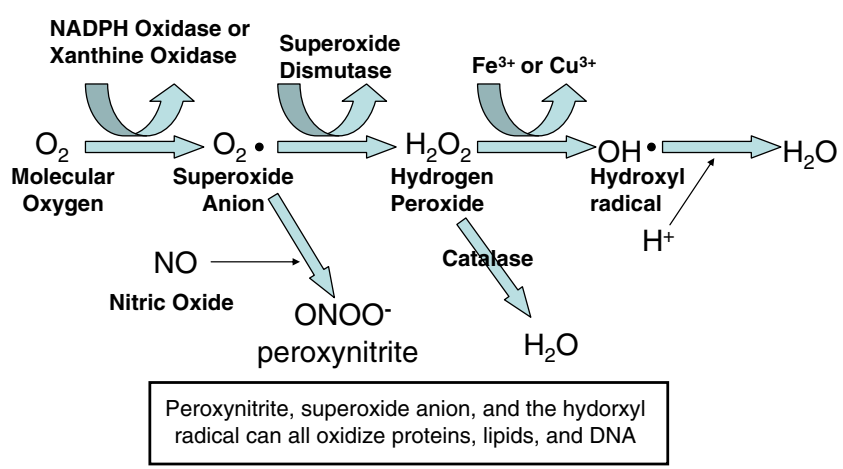

Fig. 1 Schematic representation of the pathway of radical generation and oxidative stress. $\mathrm{NADPH}=$ nicotinamide adenine dinucleotide phosphate capacity, measurement of DNA damage using chromatography, or detection of oxidized biological markers of OS. Assays for possible markers of OS measurement include measurements of oxidation of lipids, such as lipid hydroperoxides, thiobarbituric acid reacting substances (TBARS), in particular malondialdehyde, and isoprostanes (free-radical induced products of neuronal arachadonic acid peroxidation); proteins, such as protein carbonyls, various tyrosine products, and methionine sulfoxidation; and DNA, such as 8-hydroxydeoxyguanosine [8-OH-dG] and Pyrimido[1,2-a]-purin-10(3H)-one [M1G]). However, none of these assays is perfect. Each has limitations regarding sensitivity, specificity, and timing of analysis [12]. Ongoing research to determine the sensitivity and specificity of these OS biomarkers in rodents, nonhuman primates, and humans resulting from multiple types of oxidative insults and to understand the relationships between various markers, should be useful for choosing an appropriate OS marker in a given study in the future.

The use of cerebral microdialysis to sample extracellular brain fluid has been used to study free radicals and OS in traumatic brain injury, in both animals and humans [13]. This technique allows for real-time biochemical monitoring of markers of ischemia and neuronal injury, and is being increasingly used in highly advanced neurocritical care units. It certainly holds promise as a tool to improve our understanding of OS and its management in the neurocritical care setting, and for future use as a means for direct administration of potential antioxidant therapy.

Imaging techniques hold promise as useful tools for assessment of OS in vivo, although the clinical utility of these techniques remains limited. Nuclear magnetic resonance spectroscopy, such as the Nuclear Overhauser effect enhanced magnetic resonance imaging (MRI), electron spin resonance spectroscopy, and ${ }^{31} \mathrm{P}$-nuclear magnetic resonance spectroscopy can create images of free radical distributions in animals [14, 15]. Brain damage attributed to OS may be detected on MRI. For example, the relaxivity of the pro-oxidant ferric form of hemoglobin on T1-weighted imaging is higher than its ferrous counterparts [16].

\section{Role of Oxidative Stress in Various Neurological Conditions}

Ischemic Stroke

Oxidative modifications of DNA, protein, and lipids by ROS play an important role in neuronal injury and its severity following cerebral ischemia. In a prospective study of 45 patients with acute ischemic stroke, the activities of the scavenger enzymes, SOD, and catalase were lower in 
stroke patients than controls, and the study showed a gradual increase in time for as many as 15 days poststroke, which correlated with the degree of neurological deficits [17]. In another study, plasma concentrations of $\alpha$ - and $\beta$ carotene were lower in patients with acute ischemic stroke than healthy controls, and were negatively associated with high sensitivity C-reactive protein level and the National Institute of Health Stroke Scale (NIHSS) score after adjustment for age and sex [18]. Similarly, in a study of 70 patients with acute ischemic stroke and 70 controls with similar risk factors, serum levels of NO, malondialdehyde, and glutathione were significantly elevated in stroke patients, and the levels of NO and malondialdehyde correlated with the Canadian Neurological Scale score [19]. Taffi et al. [20] investigated changes in NO and OS status in 47 acute stroke patients and age- and sex-matched healthy subjects. They found that mean plasma levels of peroxynitrite were significantly higher in stroke patients and that NO levels were associated with worsening evolution of NIHSS score from admission to day 30 , suggesting that changes in $\mathrm{NO}$ metabolism may be a marker of brain injury following ischemic stroke. However, the role of NO following ischemia is complex. Other reports have linked greater NO production to improved vascular reactivity and anti-inflammatory responses following ischemia [21, 22]. Overall, the previously described studies implicate a pathophysiological role for OS in ischemic stroke and suggest deleterious effects of OS on stroke severity and clinical outcome.

Few clinical studies examined the use of antioxidants in ischemic stroke. The ability of the free radical-trapping agent NXY-059 to reduce functional disability after ischemic stroke was previously investigated. Despite encouraging results in early phase II testing, the subsequent definitive phase III trial did not show beneficial effects [23, 24]. Several reasons have been proposed for the failure of NXY-059 [25]. Future studies investigating the role of OS on long-term poststroke recovery are needed.

Reperfusion injury plays an important role in the pathophysiology of neuronal injury following cerebral ischemia via several deleterious pathways including: cytochrome-c release, expression of matrix metalloproteinase (MMP), caspase induction, and reduction of DNA repair enzymes [26]. In a case control study of ischemic stroke patients presenting within 8 hours of stroke onset, F2-isoprostanes were elevated in stroke cases compared with controls early on, but not at later time points, and they correlated with MMP-9, indicating that OS is also implicated in activation MMP-9 and blood-brain barrier injury after ischemia reperfusion [27]. Additionally, the antioxidant enzyme SOD blocks these pathways, thereby preventing apoptosis following cerebral ischemia [28], suggesting that antioxidant therapy could be a potential therapeutic strategy to prevent reperfusion injury following reperfusion therapy, such as thrombolysis.

Intracerebral Hemorrhage

After intracerebral hemorrhage (ICH), large numbers of hemoglobin-containing red blood cells are released into the brain parenchyma. The hemoglobin released from hemolyzed red blood cells forms degradation products, including the iron-containing heme, which is metabolized by heme oxygenase to yield ferric iron $\left(\mathrm{Fe}^{+++}\right)$. Iron has been implicated in neuronal injury and delayed brain edema formation after ICH via several mechanisms including activation of lipid peroxidation, exacerbation of excitotoxicity, and inhibition of sodium-potassium pump activity [29-31]. The released free iron induces neuronal injury by catalyzing a sequence of reactions known as Haber-Weiss reaction, $\left\{\mathrm{Fe}^{++}+\mathrm{H}_{2} \mathrm{O}_{2} \rightarrow \mathrm{Fe}^{+++}+\mathrm{OH}^{\circ}+\mathrm{OH}^{\circ}\right\}$, in which superoxide and hydrogen peroxide $\left(\mathrm{H}_{2} \mathrm{O}_{2}\right)$ are converted into highly reactive toxic hydroxyl radicals $\left(\mathrm{OH}^{\circ}\right)$. Increased hydroxyl radical formation leads to OS and subsequent cell death. A large number of experimental studies show that iron is neurotoxic after ICH. Goldstein et al. [30] examined the toxicity of hemin, the ferric iron oxidation product of heme, in human neuron-like cells, and found that exposure to hemin resulted in cell death, whereas exposure to protoporphyrin IX, the iron-free congener of hemin, was not toxic, indicating that the toxicity of heme/ hemin is iron-dependent. Studies in rat models of ICH also confirm the role of iron toxicity that it is mediated via OS mechanisms. Huang et al. [32] showed an increase in brain edema and upregulation of heme oxygenase after stereotactic infusion of ferric chloride, hemoglobin, or hemin into the basal ganglia. In similar studies, Nakamura et al. [33, 34] showed an increase in $8-\mathrm{OHdG}$ in the perihematoma area after ICH or injection of ferrous iron, and that the treatment with iron chelator deferoxamine mesylate ameliorated ICH-induced changes in $8-\mathrm{OHdG}$ and increased levels of apurinic/apyrimidinic endonuclease/redox effector-factor 1 (APE/Ref-1), a protein involved in DNA repair. These findings suggest that iron-mediated OS contributes to DNA damage and brain injury after ICH and that limiting iron-mediated oxidative injury in the brain is a potential therapeutic target in ICH. Emerging evidence also links iron to neuronal injury in ICH patients. Serum ferritin on admission correlates with the relative perihematoma edema (PHE) on day 3, which coincides with the timing for hemoglobin hemolysis [35] and functional outcome at 3 months [36], and the iron content within the hematoma, estimated by MRI, correlates with PHE volume [37].

The role of OS in patients with ICH has been investigated in a small study of 13 patients with spontane- 
ous ICH and 15 patients with traumatic ICH, compared to 40 healthy controls [38]. ICH patients had significantly lower plasma levels of vitamin $\mathrm{C}$ and vitamin $\mathrm{C}$ levels inversely correlated with the severity of neurological impairment, as assessed by Glasgow Coma Scale and the NIHSS, and the diameter of the hematoma. These findings suggest depletion of antioxidants following $\mathrm{ICH}$, and also suggest that its severity, which presumably reflects the severity of OS, correlates with indices of injury-related clinical impairments. The Cerebral Hematoma and NXY Treatment Trial (CHANT) investigated the use of the free radical-trapping agent NXY-059 in ICH; treatment initiated within 6 hours of ICH onset and continued for 72 hours showed no difference in mortality or the distribution of modified Rankin Scale scores at 90 days between NXYand placebo-treated patients [39].

Clinical studies in ICH patients also have identified genome-wide associations between Apolipoprotein-E variants (APOE) $\varepsilon 2$ and $\varepsilon 4$ and lobar ICH; The APOE $\varepsilon 4$ allele amplifies the inflammatory responses, increases cerebral edema, and is associated with worse outcome after ICH $[40,41]$. In mouse models, treatment with APOE analogue, COG 1410, resulted in reduced functional deficit, decreased brain concentrations of inflammatory proteins, and less cerebral edema [42]. Clearly, more clinical studies are needed to assess the role of APOE and OS in ICH patients.

\section{Subarachnoid Hemorrhage}

In rodent models of subarachnoid hemorrhage (SAH), there is evidence of increased superoxide anion and nicotinamide adenine dinucleotide phosphate (NADPH) oxidase, both powerful oxidizing agents [43, 44]. In addition, treatment with alpha-lipoic acid, a dithiol antioxidant, improves neurological outcome and decreases brain edema and free radical generation [44]. In another study of rat model of aneurysm genesis, ROS were related to aneurysm formation [43]. In this study, rats were fed a high salt diet for 3 months in addition to a lysyl-oxidase inhibitor, an enzyme involved in catalyzing the crosslinking between collagen and elastin. Upregulation of ROS-producing genes, suppression of ROS-eliminating genes, and many highly oxidative species, such as hemeoxygenase, NOS, MMP-2, and a $47 \mathrm{kDa}$ portion of NADPH oxidase were detected within the spontaneous aneurysms that formed, using reverse transcriptase PCR analysis, Western blot, and immunohistochemistry [43]. Pre-treatment with edavarone, a free-radical scavenger, decreased size of the aneurysm and the production of the previously mentioned oxidizing agents within the aneurysm, and also within infiltrating macrophages and smooth muscle cells [43]. These effects were more profound when studying these phenomena in a p47 NADPH oxidase knockout mouse, suggesting that this may be one of the key oxidizing agents required for initiating the aneurysm signaling cascade.

The prognostic value of OS has been investigated in postsurgical aneurysmal SAH patients; cerebrospinal fluid levels of malondialdehyde emerged as significant predictors of poor outcome at 6 months [45]. A Japanese proof-of-concept study investigated the use rectal indomethicin and selective brain cooling in patients with aneurysmal $\mathrm{SAH}$ and $\mathrm{ICH}$, and found reduced cerebrospinal fluid expression of inflammatory cytokines and ROS [46]. No outcome data were presented. Few randomized, controlled clinical trials have examined the use of radical scavengers in SAH patients, but the results have been disappointing [47, 48]. The synthetic 21-aminosteroid, tirilazad mesylate, which inhibits lipid peroxidation, has been extensively studied in SAH with variable results [4951]. Overall, there was no difference in long-term outcome or symptomatic vasospasm between active treatment and placebo.

\section{Traumatic Brain Injury}

Reactive oxygen species play a role in the pathogenesis of traumatic brain injury (TBI). Cats subjected to fluid-driven piston percussion model to induce TBI have significantly more superoxide anion production after TBI compared to controls [52]. They also have persistent cerebral arteriolar dilation and reduced responsiveness to hypocapnic vasosconstriction after TBI, and the arteriolar dilation is reduced by treatment with SOD and catalase [53]. In rat models of TBI, administration of progesterone before the insult reduced levels of isoprostane and improved neurological recovery [54]. In an attempt to translate this into the clinical setting, the Progesterone for Traumatic Brain Injury: Experimental Clinical Treatment (ProTECT III), a phase III clinical study is currently enrolling patients to determine whether or not progesterone will be the first experimentally vetted treatment for TBI.

Similar to ICH, APOE is another possible target for ameliorating neurological injury after TBI. APOE has been shown to reduce ROS and other inflammatory markers after many different kinds of insults [55]. When APOE or its analogues are given to rats before TBI, they not only have improved neurological outcome, but the size of the contusion is reduced compared to controls [56]. Conversely, the APOE $\varepsilon 4$ allele, which codes for a form of APOE that is the least effective in reducing the cerebral inflammatory response, predisposes to worse outcomes after neurological injury, including TBI $[57,58]$. It remains to be seen whether APOE or its analogues have a place in the treatment of TBI. 
Organ Failure, Shock, and Cardiac Arrest

Impaired oxygen utilization at a cellular level "cytopathic hypoxia," which inhibits mitochondrial activity and results in the production of ROS and RNS, plays an important role in the propagation of multiorgan dysfunction syndrome (MODS) following shock [59]. Despite the large number of antioxidants tried in MODS, few had a significant effect on morbidity or mortality $[60,61]$. One explanation is that the antioxidants tested were not targeted to mitochondria. Examples include the cationic compound, triphenylphosphonium (TPP+), and MitoQ, an analogue of TPP+. The TPP+ accumulates within the mitochondrial preventing oxidation of any part of the electron transport chain. Injection of MitoQ in a sepsis model reduced radical production and lowered markers of hepatic and renal injury [62]. In addition, some systemic antioxidants have been tried for sepsis: N-acetylcysteine and deferoxamine [6365]. Individually, these drugs have failed to improve outcome in human trials, although the combination of these drugs has shown some promise in a cecal ligation mouse model of sepsis $[66,67]$. It has been theorized that this may be due to a much more oxidative environment in sepsis that results in all of the $\mathrm{N}$-acetylcysteine being oxidized by free iron released from cytochome-c and hemolysis. Subcutaneous injection of $\mathrm{N}$-acetylcysteine and deferoxamine (3 hours after cecal ligation) resulted in decreased cytochrome-c release, radical formation, and liver necrosis [66]. These results suggest that the complex pathway of OS-mediated injury in critically ill patients may necessitate a multimodal poly-therapeutic approach, as opposed to single therapeutic strategies.

There has been some success in the treatment of sepsis with antioxidants, namely, omega fatty acids and selenium. A German group randomized 10 septic patients requiring parenteral nutrition to receive either a standard omega 6based lipid infusion or a fish-derived omega 3 lipid infusion [68]. The neutrophils derived from the omega 3 group were better able to metabolize free fatty acids, which are potent oxidants, and responded to ex vivo stimulation more briskly. The study was too small to demonstrate any improved outcome, but the results are promising and should be investigated further. Selenium has been tested in severe sepsis, with 249 patients who had APACHE III scores greater than 70 who were randomized to receive intravenous selenium for 14 days or placebo [69]. The selenium group had a significant improvement in 30-day mortality. Furthermore, the mortality benefit was accentuated in the most critically ill patients with APACHE scores greater than 105 , more than three organ failures, and those with disseminated intravascular coagulation. The investigators also measured glutathione peroxidases- 3 activity and found much higher activity in the treatment group. Both omega-3 fatty acids and selenium are relatively simple measures that could be instituted in intensive care units across the country, however, further investigation and larger studies are required before antioxidants will see their roles elevated to the standard of care for MODS.

Another type of organ failure that neurointensivists commonly deal with is cardiac arrest. Oxidative stress plays an important role in brain injury following cardiac arrest. In rats subjected to 7.5 minutes of cardiac arrest, TBARS and conjugated dienes' contents increased, respectively, by 339 and $286 \%$ compared to pre-arrest values [70]. In humans, exposure of human umbilical vein endothelial cells to the plasma of out of hospital-cardiac arrest patients increased the production of ROS and decreased production of anti-oxidants, such as SOD, glutathione, and glutathione peroxidase in the cells exposed to cardiac arrest patients vs. normal controls [71]. The protective effects of therapeutic hypothermia in cardiac arrest patients on mortality and neurological outcomes is well established [72]. Animal studies show that hypothermia decreases the production of ROS, stabilizes mitochondrial membrane potentials, phosphorlyates a survival kinase called Akt, and decreases cell death compared to normothermia in murine cardiac myocytes exposed to conditions simulating ischemia and reperfusion [73]. In rats, hypothermia reduces malondialdehyde levels in the brain during reperfusion following hypoxic ischemia compared with normothermia [74].

\section{Acute Lung Injury}

Acute lung injury (ALI) and adult respiratory distress syndrome (ARDS) are common problems in neurocritical patients. The inciting events are defined by either direct injury to the lung, such as aspiration or pneumonia versus indirect injury to the lung, such as sepsis [75]. Reactive species in ARDS come from a variety of sources including high inspired fraction of oxygen, depletion of endogenous anti-oxidants, and intra- and extra-pulmonary inflammation $[76,77]$. A study measuring oxidized products of xanthine oxidase in ARDS patients correlated strongly with outcome, supporting the role for ROS in the pathophysiology of ARDS [77].

Similar to shock studies, several anti-oxidants have been investigated for ARDS in the clinical setting; $\mathrm{N}$ acetylcysteine replenished levels of neutrophilic glutathione, but had no effect on oxidant or elastase production [77]. Another study using intravenous liposomal delivery of prostaglandin E1 to ARDS patients in order to decrease the production of exhaled hydrogen peroxide and ROS production found no effect at all on reducing oxidants or improving outcome [78]. However, mechanical ventilation with lower tidal volume than usually used appears to be an 
effective anti-oxidant strategy in patients with ALI/ARDS. In the Acute Respiratory Distress Syndrome Network (ARDSNET) trial, low tidal volumes resulted in decreased mortality for ALI/ARDS patients [79]. Animal models undergoing high tidal volume ventilation show increased expression of NOS, suggesting that this may be one of mechanisms underlying the benefits of low tidal volume in ALI/ARDS patients [80].

\section{Potential Antioxidant Strategies}

The biochemical changes of OS suggest that antioxidant therapy may be theoretically achieved by the following strategies: 1) restoring endogenous antioxidants and nutrients and supplementation with exogenous trace elements, vitamins, and nutrients with antioxidant properties; or 2) administering drugs that reduce OS, such as statins, iron-modifying agents such as deferoxamine, or minocycline. We discuss below the potential utility of these strategies and present data regarding their use in the critical care setting. It is intuitive that any of these strategies would probably be more efficacious if implemented before a massive OS takes place, i.e., before undergoing surgery or the occurrence of sepsis or organ failure. Therefore, these candidate antioxidant strategies can potentially be used for preventive or therapeutic purposes.

\section{Antioxidant Nutrients in Neurocritical Care}

There is a rationale to support exogenous supply of antioxidant trace elements, vitamins, and nutrients during critical illness. The levels of nutrients with antioxidant properties are decreased in critical illness. For example, patients with ICH have lower plasma levels of vitamin C compared with healthy volunteers [38]. Other studies have shown a reduction in muscle glutamine and glutathione and muscle protein synthesis in intensive care patients [81]. There is also evidence that low endogenous stores on antioxidant nutrients are associated with increased free radical generation, augmentation of the systemic inflammatory response, subsequent cell injury and death, and even higher morbidity and mortality in critically ill patients $[6$, 7]. In addition, proof-of-concept studies have shown that supplementation with trace elements and vitamins, such as selenium, and vitamins $\mathrm{A}, \mathrm{C}$, and $\mathrm{E}$, improve antioxidant capacity by increasing the activity of glutathione peroxidase, or reducing plasma TBARS, and isoprostanes [82]. Vitamins $\mathrm{C}$ and $\mathrm{E}$ may also reduce infectious complications by restoring neutrophil function and cell-mediated immunity, and selenium improves phagocytosis and immunoglobulin synthesis.
Supplementation of critically ill patients with antioxidant nutrients, alone or in combination, is often referred to as "immune nutrition"; and has been studied in various, double-blind, randomized, placebo-controlled trials [8385]. Although results from some of these trials were encouraging, the benefit of this therapy has not been clearly established. Several reasons have been proposed: 1) the vast majority of these studies were performed in small and heterogeneous populations of critically ill patients, and were largely underpowered; and 2) uncertainties regarding the correct dose to use, appropriate time to start immune nutrition, and the appropriate route of administration; the enteral versus parenteral. In a metaanalysis of 11 studies (selected from 44 studies based on strict criteria) of early immune nutrition using antioxidant trace elements and vitamins in 886 well-defined intensive care patient populations, Heyland et al. [86] found a significant reduction in mortality, especially with selenium alone or in combination with other micronutrients. They also found that none of the trials using immune nutrition reported deleterious effects from using exogenous micronutrients, and they concluded that supplementation with antioxidant trace elements and vitamins in intensive care patients is safe and possibly beneficial. They argued that immune nutrition must be started very early after admission to the intensive care unit and that high-dose parenteral nutrition appears to have a stronger impact on outcome. Crimi et al. [83] echoed these views and suggested that the lack of adverse effects, coupled with the minimal expense, supports the use of antioxidants in critically ill patients. Recently, Casaer et al. [87] reported that early parenteral nutrition during the first week in severely ill intensive care unit patients is associated with increased infection rates and the need for intubation compared to withholding it until day 8. It is noteworthy, however, that Casaer et al. [87] used standard parenteral nutrition preparation with low proteinto-energy ratio rather than a preparation with glutamine or other immune-modulating compounds. Given the previously described, the use of immune nutrition therapy in neurocritical care might be a rationale and promising strategy. However, more definitive evidence from largescale, randomized, placebo-controlled, and well-designed trials in this specific patient population is still required.

Recently, Manzanares and Hardy [88] argued that vitamin B12 is a "forgotten" micronutrient in critical illness, and called for well-designed clinical studies to assess the efficacy of high-dose vitamin B12 as a pharmaco-nutrient strategy to improve outcome in critically ill patients. Vitamin B12 has glutathione-sparing antioxidant properties via stimulation of methionine synthase activity and reaction with ROS and NOS. It also inhibits NOS and NO production, and decreases NF-kB activation. 


\section{Pharmacological Antioxidant Therapy}

\section{N-Acetylcysteine}

$\mathrm{N}$-acetylcysteine has multiple putative antioxidant properties by decreasing NF- $\mathrm{KB}$ activation and cytokines production, regenerating $\mathrm{NO}$ as a sulfhydryl donor, and replenishing glutathione, and scavenging ROS as a precursor of glutathione. $\mathrm{N}$-acetylcysteine has been extensively studied in critically ill patients with acute respiratory distress syndrome, organ failure, and septic shock. These studies have produced inconsistent results [83, 89, 90]. Crimi et al. [83] suggested that larger trials in specific populations of critically ill patients in which $\mathrm{N}$ acetylcysteine is administered early could potentially provide some beneficial effects. We found no studies of $\mathrm{N}$-acetylcysteine in neurocritical care, to date.

\section{Albumin}

Albumin has antioxidant effects, largely mediated by its thiol (sulfhydryl group) portion, which attaches to ROS and NOS. It also decreases copper-mediated lipid peroxidation, and modulates nitric oxide-mediated vascular dilatation. Hypoalbuminemia is common in critically ill patients, including those with TBI and various neurological conditions, and is associated with increased morbidity and mortality [91].

Studies of albumin supplementation in surgical and medical critically ill patients had inconsistent results. The Saline versus Albumin Fluid Evaluation (SAFE) study of found no advantage for albumin in comparison with saline [92], and a subsequent post-hoc subgroup analysis of patients with TBI suggested that resuscitation with albumin was associated with increased odds ratio for death [93]. A Cochrane meta-analysis found no benefit in a heterogenous group of previously published studies [94]. Powner [95] recently argued that it is important to separate data from studies regarding albumin infusions intended to restore or maintain intravascular pressure or volume goals from the potential benefit of preventing hypoalbuminemia during neurocritical care.

The use of albumin in TBI, and hypertensive and traumatic ICH has been investigated in small studies [9698]. Although the clinical results were encouraging, these studies were too small and most were nonrandomized and single arm to derive definite conclusions. Similarly, an open-label, nonrandomized, dose-escalation, safety study of $25 \%$ high-dose albumin in patients with aneurysmal subarachnoid hemorrhage was recently concluded. Preliminary results from the Treatment of Subarachnoid Hemorrhage with Human Albumin (ALISAH) study indicate that doses up to 1.25 gram per kilogram were safe, tolerable, and associated with improved outcome compared to literature-based data. Part 2 of the Albumin Therapy for Neuroprotection in Acute Ischemic Stroke (ALIAS) phase III trial is currently investigating the use of high-dose albumin therapy $(2 \mathrm{~g} / \mathrm{kg})$ in patients with acute ischemic stroke. Although recruitment in ALIAS part 1 was halted due to safety concerns, preliminary efficacy data from this cohort suggests a trend toward a favorable outcome among albumin-treated subjects [99].

\section{Statins}

Statins can modulate endogenous pro-oxidative and antioxidative systems. For example, statins dose dependently inhibit platelet-mediated low-density lipoprotein oxidation and isoprostane formation, reduce malondialdehyde, and increase SOD and glutathione peroxidase activities in erythrocytes. In a recent two-center, two-arm, randomized, open-label, controlled study of pravastatin, in intensive care patients, Makris et al. [100] reported that treatment with pravastatin significantly increased the probability of being free from ventilator-associated pneumonia and of survival. Statin therapy has also been investigated in patients with ALI; it was shown to be safe and associated with a significant decrease in bronchoalveolar lavage interleukin8, but had no other effects compared to placebo [101]. Although preclinical studies have shown benefits from statins in models of TBI and related disease processes, including cerebral ischemia, ICH, and subarachnoid hemorrhage, clinical studies have been shortcoming [102]. The use of statins in patients with aneurysmal subarachnoid hemorrhage has been investigated in several small clinical trials. A meta-analysis of four randomized controlled trials involving 190 patients revealed no significant effects on vasospasm, detected by transcranial Doppler, delayed cerebral ischemia, poor outcome, or mortality [103]. The ongoing phase III Simvastatin in Aneurysmal Subarachnoid Hemorrhage (STASH) multi-center randomized controlled phase III trial should help to further clarify the role of statins in these patients. Retrospective studies suggest that antecedent use of statins is associated with improved outcome and reduced mortality in patients with acute ischemic stroke and ICH [104, 105]. Future studies examining the role of statins as an acute treatment in PHE reduction in ICH and resultant effect on mortality and functional outcome after ischemic and hemorrhagic stroke are warranted.

\section{Iron-Modifying Agents}

Studies using iron-modifying agents in animal models of cerebral ischemia and ICH provide evidence for a potential therapeutic benefit. Tirilazad mesylate, a potent inhibitor of 
iron-dependent lipid peroxidation, attenuates neuronal necrosis and improve the neurological status in several animal models of cerebral ischemia [106]. Treatment with bipyridyl, an iron-chelating agent, significantly attenuates the development of brain edema 24 hours after the induction of ischemia [107]. Similarly, treatment with deferoxamine, another iron chelator, has been shown to reduce infarct size and improve the neurological status in rats subjected to transient middle cerebral artery occlusion [108]. Deferoxamine may also alleviate reperfusioninduced injury following ischemia. In one study, treatment with deferoxamine attenuated death rate and hemorrhagic transformation in a rat model of transient focal ischemia [109]. Treatment with deferoxamine also attenuates the production of ROS, blocks hemoglobin-mediated potentiation of glutamate neurotoxicity, reduces brain malondialdehyde content and induces recovery of sodium-potassium pump activity, and exerts diverse protective effects after experimental ICH [29, 33, 110-112]. Emerging evidence also supports a potential therapeutic role for deferoxamine following intraventricular and subarachnoid hemorrhages $[113,114]$. Deferoxamine decreases the availability of free iron for the production of hydroxyl radicals by forming a stable complex with ferric iron. However, the potential beneficial effects of deferoxamine may be only partly related to its iron chelating abilities. Deferoxamine also alters iron regulatory genes and proteins binding activity, thereby reducing cellular vulnerability to iron; prevents apoptosis induced by glutathione depletion and OS by activating a signal transduction pathway leading to activation transcription factor 1/cAMP response element-binding protein and hypoxia inducible factor, and the expression of genes known to compensate for OS; inhibits prolyl 4hydroxylase activity, which may lead to protection from OS-induced cell death; this exerts anti-inflammatory effects by stimulating cyclo-oxygenase; blocks glutamate-mediated excitotoxicity; and exerts anti-phagocytic effects [115].

Very few clinical studies have investigated the use of iron-modifying agents in stroke patients. Two multi-center trials examined the use of tirilazad in acute ischemic stroke $[116,117]$. Treatment with tirilazad $(6 \mathrm{mg} / \mathrm{kg} /$ day for 3 days) beginning at a median of 4.3 hours after stroke did not improve functional outcome after 3 months in the Randomized Trial of Tirilazad in Acute Stroke (RANTTAS) [116]. The Randomized Trial of High Dose Tirilazad in Acute Stroke (RANTTAS II) investigated a higher dose $(12.5-15 \mathrm{mg} / \mathrm{kg}$ on the first day, then 10 to $12 \mathrm{mg} / \mathrm{kg} / \mathrm{day}$ for 2 days) [117]. Although recruitment into RANTTAS II was halted prematurely after enrollment of 126 patients due to safety concerns, analysis of outcome data from the participants showed that treatment was associated with a $14 \%$ absolute reduction in mortality and an increase in the proportion of patients who were independent (Barthel Index score $>60$ ) at 3 months. The use of tirilazad mesylate in patients with subarachnoid hemorrhage was also investigated in several randomized, placebo-controlled, trials. Although it reduced the incidence of symptomatic vasospasm, it had no effect on the overall long-term clinical outcome [118]. Clinical investigations of deferoxamine in stroke have gained attention in recent years. In ICH, a small, open-label, phase-I, safety and dose-finding study of deferoxamine in patients with spontaneous ICH (safety and tolerability of deferoxamine in acute cerebral hemorrhage deferoxamine mesylate in ICH) was recently completed [119]. This study enrolled 20 subjects into 5 dose tiers of deferoxamine, which was given as a continuous intravenous infusion for 3 consecutive days starting within $16 \mathrm{~h}$ of ICH symptoms onset. Repeated daily infusions of deferoxamine were well tolerated and did not cause excessive serious adverse events or mortality. Analysis of clinical and radiological data from this study showed some encouraging trends; $50 \%$ of deferoxamine-treated patients had modified Rankin Scale (mRS) score 0 to 2 at 3 months, and deferoxamine seemed to slow down the rate of PHE progression compared with historical controls. Interestingly, deferoxamine also seemed to exert a modest blood pressure-lowering effect. Planning for a phase II trial using the identified maximum tolerated dose regimen $(62 \mathrm{mg} / \mathrm{kg} /$ day to a maximum daily dose of $6000 \mathrm{mg} /$ day) to assess the potential efficacy of deferoxamine is currently underway. Similarly, a phase I-II, double-blind, randomized, placebo-controlled, dose-finding study to evaluate the safety and pharmacokinetics of deferoxamine in patients with acute ischemic stroke who are treated with intravenous recombinant tissue plasminogen activator (rt-PA) within 3 hours of stroke onset, and to explore the effects of treatment on clinical outcomes, infarct volumes, hemorrhagic transformation, and brain edema development (The Thrombolysis and Deferoxamine in Middle Cerebral Artery Occlusion - TANDEM-1) is currently underway.

\section{Minocycline}

Minocycline has anti-inflammatory and anti-apoptotic properties, inhibits polysdenosine diphosphate ribose polymerase-1 and matrix metalloproteinases, and is an effective antioxidant and radical scavenger. Minocycline has neuroprotective effects in vivo against cerebral ischemia and in vitro against glutamate-induced cell death, an inhibition of OS by minocycline may be partly responsible for these effects [120]. Minocycline also chelates iron, and prevents the neuronal death induced by ferrous sulfate [121] and attenuated brain edema and neurological deficits in rat models of ICH [122]. An open-label, dose-escalation, safety and dose-finding study of minocycline, administered intravenously within $6 \mathrm{~h}$ of symptom onset, in patients with acute ischemic stroke was recently completed (Minocycline 
to Improve Neurologic Outcome in Stroke [MINOS]) [123], and plans for a phase III study are currently underway. Interestingly, lower plasma levels of MMP-9 was seen among rt-PA treated subjects in the MINOS trial, suggesting that combining minocycline with rt-PA might prevent the adverse consequences of thrombolysis [124].

\section{Conclusions and Future Directions}

Overwhelming pre-clinical and clinical evidence supports the presence of OS in several neurocritical conditions. Although dietary supplementation with antioxidant vitamins and the use of pharmacological agents targeting OS and its downstream cascade seems to be rational, the benefits of various attempted antioxidant strategies to date have not been clearly demonstrated. Several reasons have been advocated, including the wide variability in the nature and severity of illness in intensive care patients. Future studies should consider these issues to avoid past mistakes. Several studies of antioxidant drugs, such as statins, minocycline, and deferoxamine in specified populations of neurological patients are currently underway, and its results could have important implications in neurocritical care. Although the role of antioxidant strategies in neurocritical care is slowly evolving, it is likely to be an integral component of the overall strategy for neurological critical care in the future.

Required Author Forms Disclosure forms provided by the authors are available with the online version of this article.

\section{References}

1. Collier BR, Giladi A, Dossett LA, Dyer L, Fleming SB, Cotton BA. Impact of high-dose antioxidants on outcomes in acutely injured patients. JPEN J Parenter Enteral Nutr 2008;32:384-388.

2. Pamplona R, Costantini D. Molecular and structural antioxidant defences against oxidative stress in animals. Am J Physiol Regul Integr Comp Physiol 2011;301:R843-R863.

3. Harris RA, Amor S. Sweet and sour-oxidative and carbonyl stress in neurological disorders. CNS Neurol Disord Drug Targets 2011;10:82-107.

4. Abilés J, de la Cruz AP, Castaño J, et al. Oxidative stress is increased in critically ill patients according to antioxidant vitamins intake, independent of severity: a cohort study. Crit Care 2006;10:R146.

5. Therond P, Bonnefont-Rousselout D, Davit-Sparaul A, Conti M, Legrand A. Biomarkers of oxidative stress: an analytical approach. Curr Opin Clin Nutr Metab Care 2000;3:373-384.

6. Carré JE, Orban JC, Re L, et al. Survival in critical illness is associated with early activation of mitochondrial biogenesis. Am J Respir Crit Care Med 2010;182:745-751.

7. Gelain DP, de Bittencourt Pasquali MA, M Comim C, et al. Serum heat shock protein 70 levels, oxidant status, and mortality in sepsis. Shock 2011;35:466-470.
8. Ghosh N, Ghosh R, Mandal SC. Antioxidant protection: a promising therapeutic intervention in neurodegenerative disease. Free Radic Res 2011;45:888-905.

9. Cannizzo ES, Clement CC, Sahu R, Follo C, Santambrogio L. Oxidative stress, inflamm-aging and immunosenescence. J Proteomics 2011;74:2313-2323.

10. Altavilla D, Saitta A, Guarini S, et al. Oxidative stress causes nuclear factor-kappaB activation in acute hypovolemic hemorrhagic shock. Free Radic Biol Med 2001;30:1055-1066.

11. Szabó C. Multiple pathways of peroxynitrite cytotoxicity. Toxicol Lett 2003;140-141:105-112.

12. Grune T, Berger MM. Markers of oxidative stress in ICU clinical settings: present and future. Curr Opin Clin Nutr Metab Care 2007;10:712-717.

13. Clausen F, Marklund N, Lewén A, Enblad P, Basu S, Hillered L. Interstitial $\mathrm{F} 2$-isoprostane 8 -iso-PGF $2 \alpha$ as a biomarker of oxidative stress following severe human traumatic brain injury. J Neurotrauma 2011; doi:10.1089/neu.2011.1754.

14. Utsumi H, Yamada KI, Ichikawa K, et al. Simultaneous molecular imaging of redox reactions monitored by Overhauser-enhanced MRI with $14 \mathrm{~N}$ - and 15N-labeled nitroxyl radicals. PNAS 2006;103:1463-1468.

15. Noseworthy MD, Bray TA. Effect of oxidative stress on brain damage detected by MRI and in vivo 31P-NMR. Free Radical Biol Med 1998;6:942-951.

16. Leung G, Moody AR. MR imaging depicts oxidative stress induced by methemoglobin. Radiology 2010;257:470-476.

17. Casado A, de la Torre R, López-Fernández ME, Gil P, Egido JA. Antioxidant enzymes in acute cerebral infarction. Neurologia 2004;19:5-9.

18. Chang CY, Chen JY, Ke D, Hu ML. Plasma levels of lipophilic antioxidant vitamins in acute ischemic stroke patients: correlation to inflammation markers and neurological deficits. Nutrition 2005;21:987-993.

19. Ozkul A, Akyol A, Yenisey C, Arpaci E, Kiylioglu N, Tataroglu C. Oxidative stress in acute ischemic stroke. J Clin Neurosci 2007;14:1062-1066.

20. Taffi R, Nanetti L, Mazzanti L, et al. Plasma levels of nitric oxide and stroke outcome. J Neurol 2008;255:94-98.

21. Atochin DN, Wang A, Liu VW, et al. The phosphorylation state of eNOS modulates vascular reactivity and outcome of cerebral ischemia in vivo. J Clin Invest 2007;117:1961-1967.

22. Ishikawa $\mathrm{M}$, Zhang $\mathrm{JH}$, Nanda $\mathrm{A}$, et al. Inflammatory responses to ischemia and reperfusion in the cerebral microcirculation. Front Biosci 2004;9:1339-1347.

23. Lees KR, Zivin JA, Ashwood T, et al. NXY-059 for acute ischemic stroke. N Engl J Med 2006;354:588-600.

24. Shuaib A, Lees KR, Lyden P, et al. NXY-059 for the treatment of acute ischemic stroke. N Engl J Med 2007;357:562-571.

25. Savitz SI, Schabitz W. A Critique of SAINT II: wishful thinking, dashed hopes, and the future of neuroprotection for acute stroke. Stroke 2008;39:1389-1391.

26. Chan PH. Mitochondria and neuronal death/survival signaling pathways in cerebral ischemia. Neurochem Res 2004;29:1943-1949.

27. Kelly PJ, Morrow JD, Ning M, et al. Oxidative stress and matrix metalloproteinase-9 in acute ischemic stroke: the biomarker evaluation for antioxidant therapies in stroke (BEAT-Stroke) study. Stroke 2008;39:100-104.

28. Fujimura M, Tominaga T, Chan PH. Neuroprotective effect of an antioxidant in ischemic brain injury: involvement of neuronal apoptosis. Neurocrit Care 2005;2:59-66.

29. Regan RF, Panter SS. Hemoglobin potentiates excitotoxic injury in cortical cell culture. J Neurotrauma 1996;13:223-2231.

30. Goldstein L, Teng ZP, Zeserson E, Patel M, Regan RF. Hemin induces an iron-dependent, oxidative injury to human neuronlike cells. J Neurosci Res 2003;73:113-121. 
31. Wagner KR, Sharp FR, Ardizzone TD, Lu A, Clark JF. Heme and iron metabolism: role in cerebral hemorrhage. J Cereb Blood Flow Metab 2003;23:629-652.

32. Huang FP, Xi G, Keep RF, Hua Y, Nemoianu A, Hoff JT. Brain edema after experimental intracerebral hemorrhage: role of hemoglobin degradation products. J Neurosurg 2002;96:287-293.

33. Nakamura T, Keep RF, Hua Y, Schallert T, Hoff JT, Xi G. Deferoxamine-induced attenuation of brain edema and neurological deficits in a rat model of intracerebral hemorrhage. J Neurosurg 2004;100:672-678.

34. Nakamura T, Keep RF, Hua Y, Hoff JT, Xi G. Oxidative DNA injury after experimental intracerebral hemorrhage. Brain Res 2005;1039:30-36.

35. Mehdiratta M, Kumar S, Hackney D, et al. Association between serum ferritin level and perihematoma edema volume in patients with spontaneous intracerebral hemorrhage. Stroke 2008;39:1165-1170.

36. Pérez de la Ossa N, Sobrino T, Silva Y, et al. Iron-related brain damage in patients with intracerebral hemorrhage. Stroke 2010;41:810-813.

37. Lou M, Lieb K, Selim M. The relationship between hematoma iron content and perihematoma edema: an MRI study. Cerebrovasc Dis 2009;27:266-271.

38. Polidori MC, Mecocci P, Frei B. Plasma vitamin C levels are decreased and correlated with brain damage in patients with intracranial hemorrhage or head trauma. Stroke 2001;32:898-902.

39. Lyden PD, Shuaib A, Lees KR, et al. CHANT Trial Investigators safety and tolerability of NXY-059 for acute intracerebral hemorrhage: the CHANT Trial. Stroke 2007;38:2262-2269.

40. Biffi A, Anderson CD, Jagiella JM, et al. APOE genotype and extent of bleeding and outcome in lobar intracerebral haemorrhage: a genetic association study. Lancet Neurol 2011;10:702709.

41. James ML, Blessing R, Bennett E, et al. Apolipoprotein E modifies neurological outcome by affecting cerebral edema but not hematoma size after intracerebral hemorrhage in humans. J Stroke Cerebrovasc Dis 2009;18:144-149.

42. Laskowitz DT, Lei B, Dawson HN, et al. The apoE-mimetic peptide, COG1410, improves functional recovery in a murine model of intracerebral hemorrhage. Neurocrit Care 2011; doi:10.1007/s12028-011-9641-5.

43. Aoki T, Nishimura M, Kataoka H, Ishibashi R, et al. Reactive oxygen species modulate growth of cerebral aneurysms: a study using the free radical scavenger edaravone and p47phox $(-1-)$ mice. Lab Invest 2009;89:730-741.

44. Erşahin M, Cetinel S, Yüksel M, et al. Alpha lipoic acid alleviates oxidative stress and preserves blood brain permeability in rats with subarachnoid hemorrhage. Neurochem Res 2010;35:418-428.

45. Kaneda K, Fujita M, Yamashita S, et al. Prognostic value of biochemical markers of brain damage and oxidative stress in post-surgical aneurysmal subarachnoid hemorrhage patients. Brain Res Bull 2010;81:173-177.

46. Dohi K, Ikeda Y, Fujita S, et al. Pharmacological brain cooling with indomethacin in acute hemorrhagic stroke: antiinflammatory cytokines and antioxidative effects. Acta Neurochir Suppl 2006;96:57-60.

47. Asano T, Sano K, Kikuchi H, et al. Effects of a hydroxyl radical scavenger on delayed ischemic neurological deficits following aneurysmal subarachnoid hemorrhage: results of a multicenter, placebo-controlled double-blind trial. J Neurosurg 1996;84:792803.

48. Zuccarello M, Schmitt G. Effect of the 21-aminosteroid U74006F on cerebral vasospasm following subarachnoid hemorrhage. J Neurosurg 1989;71:98-104.

49. Kassell NF, Apperson-Hansen C, Alves WM. Randomized, double-blind, vehicle-controlled trial of tirilazad mesylate in patients with aneurysmal subarachnoid hemorrhage: a cooperative study in Europe, Australia, and New Zealand. J Neurosurg 1996;84:221-8.

50. Haley EC Jr, Apperson-Hansen C, Maile MH, et al. A randomized, double-blind, vehicle-controlled trial of tirilazad mesylate in patients with aneurysmal subarachnoid hemorrhage: a cooperative study in North America. J Neurosurg 1997;86:467-474.

51. Lanzino G, Kassell NF. Double-blind, randomized, vehiclecontrolled study of high-dose tirilazad mesylate in women with aneurysmal subarachnoid hemorrhage. Part II. A cooperative study in North America. J Neurosurg 1999;90:1018-1024.

52. Kontos HA, Wei EP. Superoxide production in experimental brain injury. J Neurosurg 1986;64:803-807.

53. Wali B, Sayeed I, Stein DG. Improved behavioral outcomes after progesterone administration in aged male rats with traumatic brain injury. Restor Neurol Neurosci 2011;29:61-71.

54. Roof RL, Hoffman SW, Stein DG. Progesterone protects against lipid peroxidation following traumatic brain injury in rats. Mol Chem Neuropathol 1997;31:1-11.

55. Poirier J. Apolipoprotein E in animal models of CNS injury and in Alzheimer's disease. Trends Neurosci 1994;17:525-530.

56. Hoane MR, Holland MA, Birky ND, Dang T, et al. The novel apolipoprotein E-based peptide COG1410 improves sensorimotor performance and reduces injury magnitude following cortical contusion injury. J Neurotrauma 2007;24:1108-1118.

57. Smith C, Graham DI, Murray LS, Stewart J, et al. Association of APOE e4 and cerebrovascular pathology in traumatic brain injury. J Neurol Neurosurg Psychiatry 2006;77:363-366.

58. Ariza M, Pueyo R, Matarín Mdel M, et al. Influence of APOE polymorphism on cognitive and behavioural outcome in moderate and severe traumatic brain injury. J Neurol Neurosurg Psychiatry 2006;77:1191-1193.

59. Fink MP. Cytopathic hypoxia. Mitochondrial dysfunction as mechanism contributing to organ dysfunction in sepsis. Crit Care Clin 2001;17:219-237.

60. Berger MM, Chiolero RL. Antioxidant supplementation in sepsis and systemic inflammatory response syndrome. Crit Care Med 2007;35:s584-s590.

61. Biesalski HK, McGregor GP. Antioxidant therapy in critical care-is the microcirculation the primary target? Crit Care Med 2007;35:s577-s583.

62. Lowes DA, Webster NR, Murphy MP, et al. The mitochondriatargeted antioxidant MitoQ protects against organ damage in a lipopolysaccharide-peptidoglycan model of sepsis. Free Radic Biol Med 2008;45:1559-1565.

63. Rank N, Haertel C, Lenhart A, Welte M, et al. N-acetylcysteine increases liver blood flow and improves liver function in septic shock patients: results of a prospective, randomized, doubleblind study. Crit Care Med 2000;28:3799-3807.

64. Spies CD, Reinhart K, Witt I, et al. Influence of N-acetylcysteine on indirect indicators of tissue oxygenation in septic shock patients: results from a prospective, randomized, double-blind study. Crit Care Med 1994;22:1738-1746.

65. Kazmierski WM, Wolberg G, Wilson JG, et al. Iron chelates bind nitric oxide and decrease mortality in an experimental model of septic shock. Proc Natl Acad Sci U S A 1996;93:9138-9141.

66. Ritter C, Andrades ME, Reinke A, et al. Treatment with Nacetylcysteine plus deferoxamine protects rats against oxidative stress and improves survival in sepsis. Crit Care Med 2004;32:342-349.

67. Zapelini PH, Cardoso MR, Ritter C, et al. Antioxidant treatment reverses mitochondrial dysfunction in a sepsis animal model. Mitochondrion 2008;8:211-218.

68. Mayer K, Fegbeutel C, Hattar K, et al. Omega-3 vs. omega-6 lipid emulsions exert differential influence on neutrophils in 
septic shock patients: impact on plasma fatty acids and lipid mediator generation. Intensive Care Med 2003;29:1472-1481.

69. Angstwurm MW, EL, Zimmermann T, et al. Selenium in Intensive Care (SIC): results of a prospective randomized, placebo-controlled, multiple-center study in patients with severe systemic inflammatory response syndrome, sepsis, and septic shock. Crit Care Med 2007;35:118-126.

70. Grieb P, Ryba MS, Debicki GS, et al. Changes in oxidative stress in the rat brain during post-cardiac arrest reperfusion, and the effect of treatment with the free radical scavenger idebenone. Resuscitation 1998;39:107-113.

71. Huet O, Dupic L, Batteux F, et al. Postresuscitation syndrome: potential role of hydroxyl radical-induced endothelial cell damage. Crit Care Med 2011;39:1712-1720.

72. Hypothermia after Cardiac Arrest Study Group. Mild therapeutic hypothermia to improve the neurologic outcome after cardiac arrest. N Engl J Med 2002;346:549-556.

73. Shao ZH, Sharp WW, Wojcik KR, et al. Therapeutic hypothermia cardioprotection via Akt- and nitric oxide-mediated attenuation of mitochondrial oxidants. Am J Physiol Heart Circ Physiol 2010;298:H2164-H2173.

74. Katz LM, Young AS, Frank JE, et al. Regulated hypothermia reduces brain oxidative stress after hypoxic-ischemia. Brain Res 2004; 1017:85-91.

75. Suntharalingam G, Regan K, Keogh BF, et al. Influence of direct and indirect etiology on acute outcome and 6-month functional recovery in acute respiratory distress syndrome. Crit Care Med 2001;29:562-566.

76. Freeman BA, Crapo JD. Biology of disease: free radicals and tissue injury. Lab Invest 1982;47:412-426.

77. Laurent T, Markert M, Feihl F, Schaller MD, et al. Oxidantantioxidant balance in granulocytes during ARDS. Effect of Nacetylcysteine. Chest 1996;109:163-166.

78. Heard SO, Longtine K, Toth I, Puyana JC, et al. The influence of liposome-encapsulated prostaglandin E1 on hydrogen peroxide concentrations in the exhaled breath of patients with the acute respiratory distress syndrome. Anesth Analg 1999;89:353-357.

79. The Acute Respiratory Distress Syndrome Network. Ventilation with lower tidal volumes as compared with traditional tidal volumes for acute lung injury and the acute respiratory distress syndrome. N Engl J Med 2000;342:1301-1308.

80. Frank JA, Pittet JF, Lee H, et al. High tidal volume ventilation induces NOS2 and impairs cAMP-dependent air space fluid clearance. Am J Physiol Lung Cell Mol Physiol 2003;284:L791L798.

81. Biolo G, Zorat F, Antonione R, et al. Muscle glutamine depletion in the intensive care unit. Int J Biochem Cell Biol 2005;37:2169-2179.

82. Crimi E, Liguori A, Condorelli M, et al. The beneficial effects of antioxidant supplementation in enteral feeding in critically ill patients: a prospective, randomized, double-blind, placebocontrolled trial. Anesth Analg 2004;99:857-863.

83. Crimi E, Sica V, Williams-Ignarro $\mathrm{S}$, et al. The role of oxidative stress in adult critical care. Free Radic Biol Med 2006;40:398-406.

84. Andrews PJD, Avenell A, Noble DW, et al. Scottish Intensive Care Glutamine or Selenium Evaluative Trial Trials Group. Randomised trial of glutamine, selenium, or both, to supplement parenteral nutrition for critically ill patients. BMJ 2011;342: d1542.

85. Berger MM, Soguel L, Shenkin A, et al. Influence of early antioxidant supplements on clinical evolution and organ function in critically ill cardiac surgery, major trauma, and subarachnoid hemorrhage patients. Crit Care 2008;12:R101.

86. Heyland DK, Dhaliwal R, Suchner U, Berger MM. Antioxidant nutrients: a systematic review of trace elements and vitamins in the critically ill patient. Intensive Care Med $2005 ; 31: 327-337$.
87. Casaer MP, Mesotten D, Hermans G, et al. Early versus late parenteral nutrition in critically Ill adults. New Engl J Med 2011;365:506-517.

88. Manzanares W, Hardy G. Vitamin B12: the forgotten micronutrient for critical care. Curr Opin Clin Nutr Metab Care 2010;13:662-668.

89. Heller AR, Groth G, Heller SC, et al. N-acetylcysteine reduces respiratory burst but augments neutrophil phagocytosis in intensive care unit patients. Crit Care Med 2001;29:272-276.

90. Molnár Z, Shearer E, Lowe D. N-Acetylcysteine treatment to prevent the progression of multisystem organ failure: a prospective, randomized, placebo-controlled study. Crit Care Med 1999;27:1100-1104.

91. Sung J, Bochicchio GV, Joshi M, et al. Admission serum albumin is predicitve of outcome in critically ill trauma patients. Am Surg 2004;70:1099-1102.

92. Finfer S, Bellomo R, Boyce N, French J, Myburgh J, Norton R; SAFE Study Investigators. A comparison of albumin and saline for fluid resuscitation in the intensive care unit. N Engl J Med 2004;350:2247-2256.

93. SAFE Study Investigators; Australian and New Zealand Intensive Care Society Clinical Trials Group; Australian Red Cross Blood Service; George Institute for International Health, Myburgh J, Cooper DJ, Finfer S, Bellomo R, Norton R, Bishop N, Kai Lo S, Vallance S. Saline or albumin for fluid resuscitation in patients with traumatic brain injury. N Engl J Med 2007;357(9):874-884.

94. Perel P, Roberts I. Colloids versus crystalloids for fluid resuscitation in critically ill patients. Cochrane Database Syst Rev 2011;(3):CD000567.

95. Powner DJ. In my opinion: serum albumin should be maintained during neurocritical care. Neurocrit Care 2011;14:482-488.

96. Jungner M, Grände PO, Mattiasson G, et al. Effects on brain edema of crystalloid and albumin fluid resuscitation after brain trauma and hemorrhage in the rat. Anesthesiology 2010;112:1194-1203.

97. Tone $\mathrm{O}$, Ito $\mathrm{U}$, Tomita $\mathrm{H}$, et al. High colloid oncotic therapy for brain edema with cerebral hemorrhage. Acta Neurochir Suppl (Wien) 1994;60:568-570.

98. Rodling Wahlström M, Olivecrona M, Nyström F, Koskinen LO, et al. Fluid therapy and the use of albumin in the treatment of severe traumatic brain injury. Acta Anaesthesiol Scand 2009;53:18-25.

99. Hill MD, Martin RH, Palesch YY, et al; ALIAS Investigators; Neurological Emergencies Treatment Trials Network. The Albumin in Acute Stroke Part 1 Trial: an exploratory efficacy analysis. Stroke 2011;42:1621-1625.

100. Makris D, Manoulakas E, Komnos A, et al. Effect of pravastatin on the frequency of ventilator-associated pneumonia and on intensive care unit mortality: Open-label, randomized study. Crit Care Med 2011;39:2440-2446.

101. Craig TR, Duffy MJ, Shyamsundar M, et al. A randomized clinical trial of hydroxymethylglutaryl- coenzyme a reductase inhibition for acute lung injury (The HARP Study). Am J Respir Crit Care Med 2011;183:620-626.

102. Wible EF, Laskowitz DT. Statins in traumatic brain injury. Neurotherapeutics 2010;7:62-73.

103. Vergouwen MD, de Haan RJ, Vermeulen M, et al. Effect of statin treatment on vasospasm, delayed cerebral ischemia, and functional outcome in patients with aneurysmal subarachnoid hemorrhage: a systematic review and meta-analysis update. Stroke 2010;41:47-52.

104. Biffi A, Devan WJ, Anderson CD, et al. Statin treatment and functional outcome after ischemic stroke: case-control and metaanalysis. Stroke 2011;42:1314-1319.

105. Biffi A, Devan WJ, Anderson CD, et al. Statin use and outcome after intracerebral hemorrhage: case-control study and metaanalysis. Neurology 2011;76:1581-1588. 
106. Feng Y, LeBlanc MH, LeBlanc EB, et al. Desmethyl tirilazad improves neurologic function after hypoxic ischemic brain injury in piglets. Crit Care Med 2000;28:1431-1438.

107. Oubidar M, Boquillon M, Marie C, et al. Ischemia-induced brain iron delocalization: effect of iron chelators. Free Radic Biol Med 1994;16:861-867.

108. Hanson LR, Roeytenberg A, Martinez PM, et al. Intranasal deferoxamine provides increased brain exposure and significant protection in rat ischemic stroke. J Pharmacol Exp Ther 2009;330:679-686.

109. Xing Y, Hua Y, Keep RF, et al. Effects of deferoxamine on brain injury after transient focal cerebral ischemia in rats with hyperglycemia. Brain Res 2009;1291:113-121.

110. Bilgihan A, Turkozkan N, Aricioglu A, et al. The effect of deferoxamine on brain lipid peroxide levels and Na-K ATPase activity following experimental subarachnoid hemorrhage. Gen Pharmacol 1994;25:495-497.

111. Gu Y, Hua Y, Keep RF, et al. Deferoxamine reduces intracerebral hematoma-induced iron accumulation and neuronal death in piglets. Stroke 2009;40:2241-2243.

112. Okauchi M, Hua Y, Keep RF, et al. Effects of deferoxamine on intracerebral hemorrhage-induced brain injury in aged rats. Stroke 2009;40:1858-1863.

113. Chen Z, Gao C, Hua Y, et al. Role of iron in brain injury after intraventricular hemorrhage. Stroke 2011;42:465-470.

114. Lee JY, Keep RF, He Y, et al. Hemoglobin and iron handling in brain after subarachnoid hemorrhage and the effect of deferoxamine on early brain injury. J Cereb Blood Flow Metab 2010;30:1793-1803.
115. Selim M. Deferoxamine mesylate: a new hope for intracerebral hemorrhage: from bench to clinical trials. Stroke 2009;40(3 Suppl):S90-S91.

116. A randomized trial of tirilazad mesylate in patients with acute stroke (RANTTAS). The RANTTAS Investigators. Stroke 1996;27:1453-1458.

117. Haley EC Jr. High-dose tirilazad for acute stroke (RANTTAS II). RANTTAS II Investigators. Stroke 1998;29:1256-1257.

118. Jang YG, Ilodigwe D, Macdonald RL. Meta-analysis of tirilazad mesylate in patients with aneurysmal subarachnoid hemorrhage. Neurocrit Care 2009;10:141-147.

119. Selim M, Yeatts S, Goldstein J, et al. Safety and tolerability of deferoxamine mesylate in patients with acute intracerebral hemorrhage. Stroke 2011;42:3067-3074.

120. Morimoto N, Shimazawa M, Yamashima T, Nagai H, Hara H. Minocycline inhibits oxidative stress and decreases in vitro and in vivo ischemic neuronal damage. Brain Res 2005;1044:8-15.

121. Chen-Roetling J, Chen L, Regan RF. Minocycline attenuates iron neurotoxicity in cortical cell cultures. Biochem Biophys Res Commun 2009;386:322-326.

122. Wu J, Yang S, Hua Y, Liu W, Keep RF, Xi G. Minocycline attenuates brain edema, brain atrophy and neurological deficits after intracerebral hemorrhage. Acta Neurochir Suppl 2010;106:147-150.

123. Fagan SC, Waller JL, Nichols FT, et al. Minocycline to improve neurologic outcome in stroke (MINOS): a dose-finding study. Stroke 2010;41:2283-2287.

124. Switzer JA, Hess DC, Ergul A, et al. Matrix Metalloproteinase-9 in an exploratory trial of intravenous minocycline for acute ischemic stroke. Stroke 2011;42:2633-2635. 